\title{
Understanding multifactorial influences on the continuum of maternal weight trajectories in pregnancy and early postpartum: study protocol, and participant baseline characteristics
}

Tiffany A Moore Simas ${ }^{1 *}$, Silvia Corvera ${ }^{2}$, Mary M Lee ${ }^{3}$, NingNing Zhang ${ }^{3}$, Katherine Leung ${ }^{1}$, Barbara Olendzki ${ }^{4}$, Bruce Barton ${ }^{5}$ and Milagros C Rosal ${ }^{4}$

\begin{abstract}
Background: Maternal and offspring immediate and long-term health are affected by pregnancy weight gain and maternal weight. This study was designed to determine feasibility of: 1) recruiting a socio-economically and racially/ethnically diverse sample of pregnant women into a longitudinal observational study, including consenting the women for serial biologic specimen evaluations; 2) implementing comprehensive assessments (including biologic, anthropometric, behavioral, cognitive/psychosocial and socio-demographic, and cultural measures) at multiple time points over the study period, including collecting biologic specimens at planned and unplanned pregnancy delivery times; and 3) retaining the sample for one year into the postpartum period. Additionally, the study will provide preliminary data of associations among hypothesized predictors, mediators and moderators of pregnancy and post-partum maternal and infant weight trajectories. The study was conceptualized under a Biopsychosocial Model using a lifespan approach. Study protocol and baseline characteristics are described.

Methods/Design: We sought to recruit a sample of 100 healthy women age 18-45 years, between 28-34 weeks gestation, with singleton pregnancies, enrolled in care prior to 17 weeks gestation. Women provide written consent for face-to-face (medical history, anthropometrics, biologic specimens), and paper-and-pencil assessments, at five time points: baseline (third trimester), delivery-associated, and 6-weeks, 3-months and 6-months postpartum. Additional telephone-based assessments (diet, physical activity and breastfeeding) administered baseline and three-months postpartum. Infant weights are collected until 1-year of life. We seek to retain $80 \%$ of participants at six-months postpartum and $80 \%$ of offspring at 12 -months.

110 women were recruited. Sample characteristics include: mean age 28.3 years, BMI $25.7 \mathrm{~kg} / \mathrm{m}^{2}$, and gestational age at baseline visit of 32.5 weeks. One-third of cohort was non-white, over a quarter were Latina, and almost a quarter were non-US born. The cohort majority was multigravida, had graduated high school and/or had higher levels of education, and worked outside the home.
\end{abstract}

Discussion: Documentation of study feasibility and preliminary data for theory-driven hypothesis of maternal and child factors associated with weight trajectories will support future large scale longitudinal studies of risk and protective factors for maternal and child health. This research will also inform intervention targets facilitating healthy maternal and child weight.

Keywords: Design, Methods, Biopsychosocial, Pregnancy, Postpartum, Weight gain, Weight loss, Weight retention, Maternal and child health, Well being

\footnotetext{
* Correspondence: TiffanyA.MooreSimas@UMassMemorial.org

'Department of Obstetrics \& Gynecology, Division of Research, University of

Massachusetts Medical School/UMass Memorial Health Care, Memorial

Campus - 119 Belmont Street, Worcester, MA 01605, USA

Full list of author information is available at the end of the article
} 


\section{Background}

Maternal weight before, during, and after pregnancy is of considerable public health importance given its impact on both immediate and long-term maternal and child health. The majority of women and thus their children are at weight-related health risk when considering: (1) two-thirds of American women are overweight or obese [1-4], with nearly $50 \%$ entering pregnancy overweight or obese, and one in five obese [5], (2) up to $84 \%$ of overweight and $74 \%$ of obese pregnant women gain above recommended guidelines [6], (3) up to two thirds of women retain weight after pregnancy [7], and (4) some women continue to gain weight rather than lose weight in the postpartum period [8]. Excessive gestational weight gain contributes to subsequent postpartum weight retention [7], and failure to lose pregnancy weight by six months postpartum predicts long-term obesity $[9,10]$ with women retaining an average of $3 \mathrm{~kg}$ per pregnancy at 10 years [11]. Given well-established associations between obesity and an array of chronic maternal health conditions, including cardiometabolic diseases [12-15] and some cancers [16], achieving a healthy weight gain during pregnancy, and preventing postpartum weight retention, are critical to the long-term health of many women $[9,10]$.

Maternal obesity is associated with increased risks of gestational diabetes, large for gestational age neonates, and childhood obesity [17]. Excessive maternal weight gain in pregnancy is also associated with childhood obesity [18] which is subsequently associated with longterm offspring consequences including cardiometabolic and neuro-developmental disorders. The growing body of evidence in support of the Developmental Origins of Health and Disease Hypothesis (DOHaD, the Barker hypothesis) [19] suggests that optimizing maternal health before and during pregnancy is critical for improved outcomes not just for women but also for their children.

Acting upon scientific literature on weight gain patterns before, during, and after pregnancy, and in the context of a life-stage framework [3], the Institute of Medicine (IOM) issued gestational weight gain recommendations in 1999, with revisions in 2009. Current recommendations encourage appropriate gain for women with underweight BMIs $\left(<18.5 \mathrm{~kg} / \mathrm{m}^{2}\right)$ of $28-40$ pounds, normal BMIs $\left(18.5-24.9 \mathrm{~kg} / \mathrm{m}^{2}\right)$ of $25-35$ pounds, overweight BMIs $\left(25.0-29.9 \mathrm{~kg} / \mathrm{m}^{2}\right)$ of $15-25$ pounds, and obese BMIs $\left(\geq 30 \mathrm{~kg} / \mathrm{m}^{2}\right)$ of $11-20$ pounds [3]. The 2009 IOM report also identified a set of consequences that were potentially causally related to gestational weight gain with postpartum weight retention and cesarean delivery emerging as being most important for maternal health; and small for gestational age neonates, large for gestational age neonates, preterm birth and childhood obesity emerging as the most important consequences for infant health [3].
In an effort to promote these guidelines, several randomized control trials have tested interventions to optimize gestational weight gain; however, such interventions have had limited effectiveness [20-26]. Similarly, randomized intervention trials for post-partum weight loss have been limited in their effectiveness [24,27]. Studies have predominantly intervened on diet and exercise, and evaluated effects of breastfeeding [28-31]. With few exceptions [32], the interventions have lacked theoretical grounding and have been limited in the potential array of variables investigated.

The general non-pregnant obesity and weight gain literature has broad comprehensive models for understanding weight and obesity. For example, there has been increased attention to the roles and interplay of reduced sleep [33-35], increased stress [36-38] and depression, all factors that fluctuate during pregnancy and the post-partum period [39-42]. However, examination of these factors has received limited research attention with regards to pregnancy and post-partum weight. In addition to psychosocial factors, biologic considerations include differences in the deposition and mobilization of peripheral and central subcutaneous adipose tissue in the non-pregnant, pregnant and lactating states [43-46].

Given inadequate literature in these and relevant areas, action items in the 2009 IOM report included a recommendation to conduct comprehensive studies that examine how dietary intake, physical activity, dieting practices, food insecurity and, more broadly, the social, cultural and environmental context affect gestational weight gain [3] in large and diverse populations of women. Gestational weight gain and postpartum weight are part of a continuum that needs to be considered in identifying risk factors and mechanisms of postpartum weight retention. Such knowledge is critical to the design of future interventions that have the potential to substantially improve maternal and consequently, child health.

A lifespan approach to the Biopsychosocial Model [47] provides a comprehensive template for examining how the complex interactions among an individual's biological, psychological and contextual factors affect weight and health outcomes during the childbearing years and beyond, for both the mother and the child. In the current study we conceptualize women's weight trajectory during both the pregnancy and postpartum periods in addition to long-term maternal and child health and well-being within this biopsychosocial framework (Figure 1). Our study was designed to understand multifactorial influences on maternal weight trajectories that begin in pregnancy and extend into the postpartum period.

\section{Study aims}

To establish the feasibility of: 1) recruiting a socioeconomically and racially/ethnically diverse sample of 


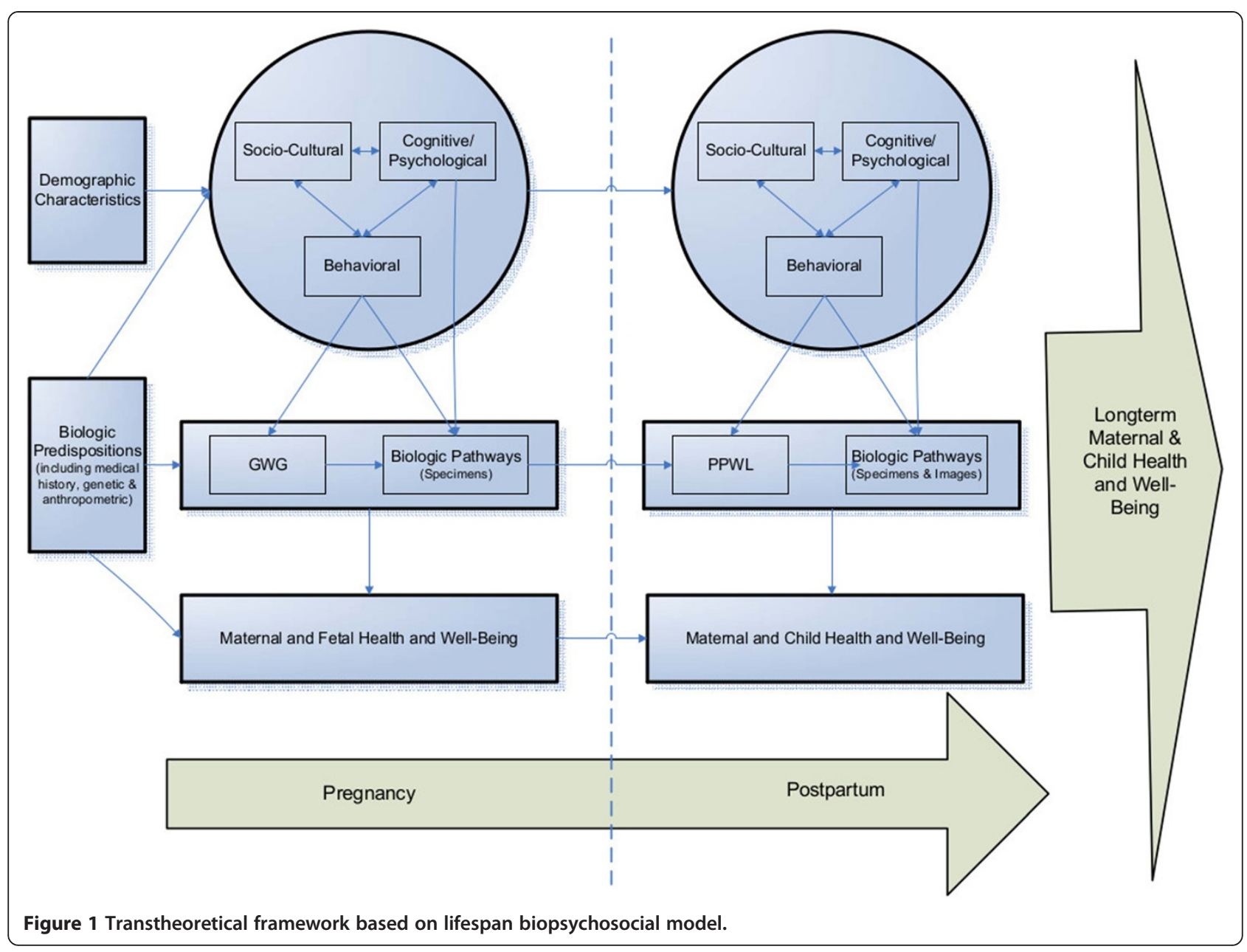

pregnant women into a longitudinal observational study, including consenting the women for serial biologic specimen evaluations; 2) implementing comprehensive assessments (including biologic, anthropometric, behavioral, cognitive/psychosocial and socio-demographic and cultural measures) at multiple time points over the study period, including collecting biologic specimens at planned and unplanned pregnancy delivery times; and 3) retaining the sample for one year into the postpartum period. Additionally, the study will provide preliminary data of associations among hypothesized predictors, mediators and moderators of pregnancy weight gain and postpartum weight loss and retention, in accordance with the multifactorial model shown in Figure 1. Ultimately, this research will inform a future larger study of risks and protective factors for gestational weight gain and post-partum weight loss, with effective interventions to optimize both. This paper describes the study design, assessment protocols, and baseline demographic characteristics of 110 diverse pregnant women and their babies.

\section{Methods}

\section{Study design \& overview}

The Pregnancy and Postpartum Observational Determinants Study (PPODS) is a prospective cohort feasibility study approved by the University of Massachusetts Medical School Institutional Review Board human subjects committee. In accordance with the Lifespan Biopsychosocial Model used as the framework for this study, comprehensive assessments starting in pregnancy and continuing over the postpartum period include demographic, biologic including anthropometric, behavioral, cognitive/psychological and socio-cultural factors hypothesized to influence gestational weight gain (GWG), postpartum weight loss, and clinical outcomes of healthy women and their offspring. At present, study recruitment is complete and participant follow up and analyses are ongoing.

\section{Study setting and population}

The study is being conducted at the ambulatory faculty and resident obstetrical practices at a large tertiary hospital in 
Central Massachusetts, UMass Memorial Medical Center (UMMMC), in Worcester, MA, where approximately 20 residents, 3 nurse practitioners, and 14 attending faculty obstetricians provide prenatal and postpartum care to ethnically and socioeconomically diverse women. These practices deliver $\sim 1,600$ of the $\sim 4,000$ pregnancies that are admitted to UMMMC each year.

Eligibility criteria include: (1) age $\geq 18$ or $\leq 45$ years, (2) singleton gestation, (3) English speaking, (4) no history of pre-gestational or current gestational diabetes, (5) no evidence of alcohol or substance abuse, (6) not taking medications that affect weight (anti-hypertensives, hypoglycemics, steroids, second generation anti-psychotics, anti-epileptics and thyroid-related pharmaceuticals), (7) no evidence of HIV, hepatitis, autoimmune disease, eating disorder history, or bariatric surgery history, and (8) prenatal care initiated $\leq 16$ weeks and 6 days gestational age. Age of inclusion was restricted as GWG recommendations are somewhat controversial in adolescents and general weight gain increases with advancing age. Multiple gestations were excluded due to differences in GWG recommendations for twins, triplets and higher order multiples. Non-English speaking subjects were excluded due to limited resources. Subjects with diabetes were ineligible due to high potentiality of receiving treatments that affect weight (i.e., selected medications, dietary interventions). Subjects with substance abuse history, with prescriptions for aforementioned medication groups and with disease states listed were excluded due to effects on weight, weight gain and diet and on potential compliance and reliability in the former population. Subjects had to have initiated care in early pregnancy so that weight measurements throughout pregnancy were available for analyses.

\section{Participant screening and recruitment}

Identification of eligible women occurred proactively. Between 24-28 weeks gestation, obstetric providers at study practices carry out universal gestational diabetes screening in all gravidas without pre-gestational type 1 or type 2 diabetes mellitus with a $50 \mathrm{~g}$ glucose load followed by plasma glucose determination in the subsequent hour [48]. For tracking purposes, a list of consecutive patients undergoing gestational diabetes screening is generated real-time; this prompted review of associated laboratory results by a trained and supervised study coordinator using a HIPAA waiver and the clinical care team. The study coordinator then reviewed medical records of women without gestational diabetes for additional study inclusion/exclusion criteria. Women meeting study criteria who had an upcoming appointment at a gestational age $<34$ weeks were placed on a list of potentially eligible women. Their health care providers were subsequently contacted for permission to approach the women and then a letter was mailed to inform potential participants about the study and that they would be approached at their next face-to-face prenatal visit. To support study recruitment efforts, advertisements were placed in public waiting rooms and examination rooms of the obstetric practices, and their physicians were also encouraged to refer patients. All eligible women who expressed interest then provided written informed consent and were assigned a unique alphanumeric identifier.

\section{Measurements and assessments}

Women were assessed at baseline, at delivery, in the immediate in-patient postpartum period and then postpartum at 6 weeks, 3 months and 6 months. Assessments included demographic, biologic including anthropometric, behavioral, cognitive/psychological and sociocultural evaluations. See Table 1 for the assessment schedule. In recognition of participants' time commitments, women receive gift card incentives following each assessment. Data collection time points for infants are birth, 3-months, 6 months and at 1 year of age. Additionally, infant weight data are obtained from their pediatricians until one year of life.

\section{Demographic}

Baseline demographic data are collected on the day of enrollment by study personnel through personal interview. Demographic factors of interest include age, race/ ethnicity, educational attainment, marital status, occupation, work status, insurance type, household size and income and other housing-related factors. Demographics with potential for change over time are queried at each postpartum visit.

\section{Biological (Including Medical History, Clinical Assessments, Anthropometric Data and Genetics)}

Baseline medical history is collected on the day of enrollment by study personnel through personal and medical record review. Characteristics surveyed include age at menarche, parity, interval from last pregnancy and obstetric history including prior complications like gestational diabetes, hypertensive disorders of pregnancy, prolonged bedrest and others. Medical chart review is performed to confirm or clarify aforementioned information and to retrieve information on medication use, pregnancy dating, and weight-related services such as nutrition consultations. Estimated delivery date and thus gestational age is determined by chart review, based on 1 st trimester ultrasound or clinical dating that agrees with 2nd trimester ultrasound [49].

Blood pressure is measured three times in the right arm after 15 mins of sitting [50] and with a 15 minute lapse between each measurement, using an automated Dinamap XL blood pressure monitor [51]. Urine is evaluated for the presence of protein or glucose using Siemens multistix 10SG reagent strips for urinalysis. 
Table 1 Schedule of study assessments

\begin{tabular}{|c|c|c|c|c|c|c|c|c|}
\hline \multirow[t]{3}{*}{ Study assessments } & & \multicolumn{7}{|c|}{ Study time points } \\
\hline & & \multirow[t]{2}{*}{ Baseline* } & \multirow[t]{2}{*}{ Delivery } & \multicolumn{5}{|c|}{ Postpartum } \\
\hline & & & & $0-4 d$ & 6 wks & $3 \mathrm{mos}$ & $6 \mathrm{mos}$ & 1 year \\
\hline \multicolumn{2}{|c|}{ Review study protocol \& informed consent } & $\checkmark$ & & & & & & \\
\hline \multicolumn{9}{|c|}{ Confirmation inclusion/exclusion criteria } \\
\hline \multicolumn{2}{|l|}{ Demographics } & $\checkmark$ & & & & & & \\
\hline \multirow[t]{8}{*}{ Biological } & Medical History & $\checkmark$ & & & & & & \\
\hline & Blood Pressure & $\checkmark$ & & $\checkmark$ & $\checkmark$ & $\checkmark$ & $\checkmark$ & \\
\hline & Urine for protein and glucose & $\checkmark$ & & $\checkmark$ & $\checkmark$ & $\checkmark$ & $\checkmark$ & \\
\hline & Height & $\checkmark$ & & & & & & \\
\hline & Prepregnancy Weight \& BMI & $\checkmark$ & & & & & & \\
\hline & Skin Fold Thickness (SFT) & $\checkmark$ & & $\checkmark$ & $\checkmark$ & $\checkmark$ & $\checkmark$ & \\
\hline & Waist, Hip and Arm Circumferences & $\checkmark$ & & $\checkmark$ & $\checkmark$ & $\checkmark$ & $\checkmark$ & \\
\hline & $\begin{array}{l}\text { Genetics (Maternal buccal swab, umbilical } \\
\text { cord and blood, placenta, adipose tissue })^{\dagger}\end{array}$ & & $\checkmark$ & & & & & \\
\hline \multirow[t]{6}{*}{ Behavioral } & 24 Hour Dietary and Physical Activity Recalls & $\checkmark$ & & & & $\checkmark$ & & \\
\hline & 3-Factor Eating Questionnaire R18-modified & $\checkmark$ & & $\checkmark$ & $\checkmark$ & $\checkmark$ & $\checkmark$ & \\
\hline & Food Craving Inventory & $\checkmark$ & & $\checkmark$ & $\checkmark$ & $\checkmark$ & $\checkmark$ & \\
\hline & $\begin{array}{l}\text { Pregnancy and Physical Activity Questionnaire } \\
\text { (PPAQ) (modified postpartum) }\end{array}$ & $\checkmark$ & & $\checkmark$ & $\checkmark$ & $\checkmark$ & $\checkmark$ & \\
\hline & Breastfeeding-Infant Feeding Surveys & & & $\checkmark$ & $\checkmark$ & $\checkmark$ & $\checkmark$ & $\checkmark$ \\
\hline & Pittsburgh Sleep Quality Index & $\checkmark$ & & & $\checkmark$ & $\checkmark$ & $\checkmark$ & \\
\hline \multirow[t]{6}{*}{ Cognitive/psychological } & Spielberger Trait Anxiety Inventory & $\checkmark$ & & $\checkmark$ & $\checkmark$ & $\checkmark$ & $\checkmark$ & \\
\hline & Perceived Stress Scale & $\checkmark$ & & $\checkmark$ & $\checkmark$ & $\checkmark$ & $\checkmark$ & \\
\hline & Quality of Life SF-12 with RAND score & $\checkmark$ & & $\checkmark$ & $\checkmark$ & $\checkmark$ & $\checkmark$ & \\
\hline & Happiness Scale & $\checkmark$ & & $\checkmark$ & $\checkmark$ & $\checkmark$ & $\checkmark$ & \\
\hline & Edinburgh Postnatal Depression Scale & $\checkmark$ & & $\checkmark$ & $\checkmark$ & $\checkmark$ & $\checkmark$ & \\
\hline & $\begin{array}{l}\text { Pregnancy \& Weight Gain Attitude } \\
\text { Scale (PWGAS), modified }\end{array}$ & $\checkmark$ & & & & & & \\
\hline \multirow[t]{3}{*}{ Socio-cultural } & $\begin{array}{l}\text { Weight gain advice and purposeful } \\
\text { weight control attempts survey }\end{array}$ & $\checkmark$ & & & $\checkmark$ & $\checkmark$ & $\checkmark$ & \\
\hline & Social Support & $\checkmark$ & & $\checkmark$ & $\checkmark$ & $\checkmark$ & $\checkmark$ & \\
\hline & Life Events & $\checkmark$ & & $\checkmark$ & $\checkmark$ & $\checkmark$ & $\checkmark$ & \\
\hline \multirow{7}{*}{$\begin{array}{l}\text { Biologic pathways } \\
\text { (specimens \& imaging) }\end{array}$} & Maternal Blood & $\checkmark$ & $\checkmark$ & $\checkmark$ & $\checkmark$ & $\checkmark$ & $\checkmark$ & \\
\hline & Umbilical Cord Blood & & $\checkmark$ & & & & & \\
\hline & Placenta & & $\checkmark$ & & & & & \\
\hline & Umbilical Cord & & $\checkmark$ & & & & & \\
\hline & Adipose Tissue ${ }^{\dagger}$ (Subcutaneous \& Omental) & & $\checkmark$ & & & & & \\
\hline & Magnetic Resonance Imaging ${ }^{\dagger}$ & & & $\checkmark$ & & & & \\
\hline & Mouthwash Buccal Epithelial Cells for DNA & $\checkmark$ & & & & & $\checkmark$ & \\
\hline \multirow[t]{2}{*}{ Weight outcomes } & Maternal Weight & $\checkmark$ & $\checkmark$ & $\checkmark$ & $\checkmark$ & $\checkmark$ & $\checkmark$ & \\
\hline & Neonatal Weight \& Length & & $\checkmark$ & $\checkmark$ & $\checkmark$ & $\checkmark$ & $\checkmark$ & $\checkmark$ \\
\hline
\end{tabular}

*Baseline visit occurring in pregnancy at approximately 28-34 weeks gestation.

${ }^{\dagger}$ Subset of subjects only.

${ }^{\ddagger}$ Participation in biologic specimen component of study was optional. 
Height is measured using a 1 Seca 213 Portable Stadiometer. Prepregnancy weight is self-reported at first prenatal visit and abstracted from prenatal record. Prepregnancy body mass index (BMI) calculated as prepregnancy weight $(\mathrm{kg}) /$ height $^{2}$ (meters $\left.^{2}\right)$. BMI considered continuously and categorically as per World Health Organization criteria: underweight $\left(<18.5 \mathrm{~kg} / \mathrm{m}^{2}\right)$, nor-

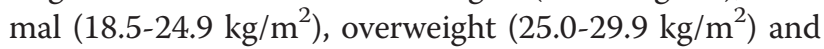
obese $\left(\geq 30 \mathrm{~kg} / \mathrm{m}^{2}\right)$ [3]. In accordance with prenatal care standard, weight and gestational age at each visit is recorded from digital clinical scales and was abstracted from records.

Skinfold thickness (SFT) measurements are performed at seven body sites (biceps, triceps, subscapular, iliac, costal, mid thigh, lower thigh), on the subjects' right side with a calibrated Harpenden skinfold caliper (British Indicators, Sussex, UK), by methods and placements as described by Huston-Presley et al. [52], to quantify tissue distribution. All skinfolds are assessed three times at each study time point; a mean value of the three is computed. In cases where two measurements differ by more than $1.0 \mathrm{~mm}$, the skin fold is measured a fourth time and the mean value of the four values is averaged.

Body circumferences (upper arm, waist and hip) are obtained recognizing that waist measurements are limited in pregnancy and the immediate postpartum period. Two waist circumferences are measured: (1) midway between the lowest lateral border of the ribs and the top of the iliac crest as per WHO recommendations and (2) at the top of the iliac crest as per NIH standards, in relaxed subjects during expiration [53]. Hip circumference is measured at the maximum circumference overlying the buttocks. Arm circumference measured midway between the axilla and the elbow. All circumferences measured 3 times and an average calculated.

Biologic specimens are collected over the course of the study with the goal of gaining insight into biologic pathways and epigenetic signatures affected by or contributing to weight outcomes. As the main aims of this study did not focus specifically on biomarkers and biosignature evaluation, but rather the acceptability of serial biospecimens collection, subjects could opt-out of contribution in part or completely.

Maternal venipuncture for blood collection is performed at each study visit for consenting subjects. Additionally, at the time of delivery, umbilical cord blood is collected after delivery of the baby but prior to delivery of the placenta. All plasma samples are aliquoted into $1 \mathrm{ml}$ cryovials and stored at $-80^{\circ} \mathrm{C}$ until assayed with freeze/thaw cycles limited to a maximum of two. All samples are analyzed in duplicate according to manufacturer instructions by a single investigator (NNZ) on the same day to minimize day-to-day variation. Samples are measured using a commercially available MagPix Milliplex ${ }^{\curvearrowleft}$ kit (EMD Millipore) with a minimum of 100 positive beads for each assay acquired with Luminex Magpix laser-based fluorescent analytic test instrumentation (Luminex Corporation, Austin, TX). Manufacture supplied controls are used to monitor coefficients of variation $(<15 \%$ and $20 \%$ for intra-assay and inter-assay, respectively). Samples are diluted as per manufacturer instructions. Serum evaluations include adiponectin, c-reactive protein (CRP), interleukin1b, interleukin-6, interleukin-8, insulin, leptin, PAI-1 total, and TNF $\alpha$.

After delivery of the placenta, a $1 \times 1 \mathrm{~cm}$ segment of placenta and a $1 \mathrm{~cm}$ length of umbilical cord are placed in $4 \%$ para-formaldehyde and a second similar sized specimen of each are placed in normal saline. Prior to study completion, a single mouthwash sample of buccal epithelial cells is collected with Scope brand mouthwash [54].

For the subset of subjects who have Cesarean delivery for obstetric indications, and who consent, two $1 \mathrm{x} 1 \mathrm{seg}-$ ments of omental (visceral adipose) and subcutaneous adipose tissue are sampled after the uterus is returned to the abdomen, and after the rectus fascia is re-approximated but prior to skin closure, respectively. From each site, one sample is placed in 4\% para-formaldehyde and one in normal saline. The size and number of adiopocytes within the samples is determined by light microscopy. Capillary density is measured by counting number of cell profiles stained with endothelial specific markers (e.g. vWF) in 10-20 fields per biopsy specimen.

To quantify adipose deposition (visceral vs. subcutaneous) following maximal GWG [55], a subset of eligible participants (whom either did not have a Cesarean delivery or whom had subcuticular stitches for skin closure at the time of Cesarean, and whom have no metal foreign bodies), are asked to undergo optional magnetic resonance imaging (MRI) during the 2-4 day postpartum inpatient stay. Images are captured centrally which decreases effect of postpartum fluid shifts that most often settle peripherally in dependent regions.

\section{Behavioral}

At each of two study time points, dietary intake is assessed using three telephone-administered 24-hour (24HR) dietary recalls (two weekdays and one weekend over a three-week window), with each call duration lasting 15-30 minutes. The first dietary assessment is conducted in pregnancy at baseline, and then again at the threemonth postpartum assessment. 24HR dietary recalls are considered the gold standard dietary assessment method for population-based research, with three recalls per time point being appropriate to examine inter- and intra- dietary variation patterns [56]. These assessments are administered by trained dieticians who elicit information on dietary intake utilizing the multiple-pass technique, allowing for several distinct opportunities to obtain information 
about participants' food intake during the previous 24 hours. This approach, and associated software, allow for range and logic error checking, information prompting and corrections. 24HR recalls are analyzed using the University of Minnesota Nutrition Coordinating Center's (NCC) Nutrition Data System for Research (NDS-R-2010) software (updated annually) [57]. The system consists of data entry and analysis software and comprehensive food nutrient databases. The database contains over 18,000 foods (including culturally unique foods) and 8,000 brand name products along with values for 155 nutrients, nutrient ratios and other food components, allowing for analysis of individual dietary variations including portion size, preparation methods, ingredients, assessments and timing of eating. A Dietary Supplement Module captures supplements used by participants.

Physical activity is assessed on the same phone call via a validated $24 \mathrm{HR}$ physical activity recall $[58,59]$. Similar to the dietary recalls, three physical activity recalls are administered at baseline (in pregnancy) and then at three months post-partum, each lasting 5-10 minutes. Detailed information about specific types and intensities of activities are summarized and metabolic equivalents (METS) are calculated using Ainsworth and colleagues compendium of physical activities [60].

Additionally, the Pregnancy and Physical Activity Questionnaire (PPAQ) [61], modified for administration in the post-partum period, is administered by research personnel at all assessment time points. The PPAQ is a self-administered measure of physical activity that has been validated using ActiGraph accelerometers. This measure provides physical activity data in various domains relevant to pregnant and postpartum women, including occupational activity, household/care-giving activities, and sports/exercise [61].

Extent of breastfeeding, breast pumping, formula and other infant feeding practices are assessed with select questions from the Center for Disease Control's (CDC) maternal-infant feeding study [62] and a modified version of the United Kingdom's Medical Research Council Epidemiology Unit's feeding and growth questionnaire [63]. Questions probe for exclusivity of breastfeeding versus extent to which other liquid and food products are offered to infants so as to quantify maternal energy expenditure with regards to lactation efforts.

Sleep quantity and quality are assessed with the 19 item Pittsburgh Sleep Quality Index [64], which has been used to assess sleep disturbance in pregnant [65] and post-partum [66] women.

Tobacco and alcohol use are queried at each study visit and recorded. Selected items from the Behavioral Risk Factor Surveillance System questionnaire [67] are utilized to assess tobacco.

\section{Cognitive/Psychological}

Self-administered survey tools are used to assess psychological and cognitive/attitudinal variables at all study time points. Areas assessed include anxiety evaluated by the 20-item Spielberger State Anxiety Inventory(SSAI)) [68], depression as measured by the the 10-item Edinburgh Postnatal Depression Scale (EPDS) [69,70], stress as assessed by the 10-item Perceived Stress Scale [71], a measure of life events [72], and quality of life assessed via the SF-12 [73]. We also assessed positive affect using the Happiness Scale modified (4 questions) [74]. Attitude towards pregnancy and weight gain were assessed using the modified Pregnancy and Weight Gain Attitude Scale (18 questions) [75] and questions on pregnancy intendedness. Selected eating behaviors namely emotional eating, uncontrolled eating and cognitive restraint are evaluated via the 18-item Three-Factor Eating Questionnaire [76], and a 28item inventory assesses food cravings [77].

\section{Sociocultural}

Sociocultural survey assessments are completed at each study time point and predominantly evaluate overall social support using the 20-item Medical Outcomes Study (MOS) social support survey [78] and pregnancy-specific social supports [79-81] (Lobel: The prenatal social support instrument (PSSI), unpublished).

\section{Weight outcomes}

Total gestational weight gain (GWG) is calculated by subtracting self-reported pre-pregnancy weight from weight at time of delivery admission or documented weight at last prenatal visit. GWG is considered continuously and categorically (inadequate, appropriate or excessive, as per IOM-recommended ranges) over the course of gestation and at delivery. Categorical GWG assessment is BMI-specific and accounts for gestational age at measurement as per IOM recommendations [82]. Minimum and maximum gain at each gestational age week is calculated based on recommended velocities of gain in first trimester (ending week 13) and throughout second and third trimester assuming the following gain ranges achieved by 40th week: $28-40 \mathrm{lbs}$ for women of underweight prepregnancy BMI, 25-35 lbs for normal, 15-25 lbs for overweight, and 11-20 lbs for obese gravidas [3].

Postpartum weight loss or retention is calculated at 6 weeks, 3 months and 6 months postpartum by subtracting measured weights at these study visits from measured weight at last prenatal care visit. All study specific weights are measured three times on a digital scale (TANITA BWB-800AS) and averaged.

Infant weights are measured in order to assess associations between maternal variables and infant weight. Infant weights are measured at all postpartum assessment 
time points when the infant is in attendance with maternal subjects, utilizing a Tanita BD-590 pediatric scale. Additionally, pediatrician records are obtained once each child reaches a year of life to capture length, weight and head circumference measurements for the offspring of each maternal participant from birth through one year.

\section{Participant safety}

To ensure the safety of participants who might be experiencing mood disorders, the EPDS is scored immediately after completion and before the participant leaves the study office. Participants scoring 12 or greater (suggestive of possible clinical depression) who score negative on the suicide question are encouraged to contact their provider to discuss symptoms. Participants scoring 12 or greater and with positive responses to the suicide question are considered at acute risk of injury or harm; an appropriately trained person performs a safety assessment and refers for immediate psychiatric evaluation if warranted.

\section{Statistical analysis, power and sample size}

The primary outcomes for this study are change in weight from pre-pregnancy to last measured prenatal weight prior to delivery (i.e. gestational weight gain or change) and change in weight from last measured prenatal weight to weight at 6 months post-partum (i.e. postpartum weight change). Additional weight measures are taken at each prenatal visit and at 6 weeks and 3 months postpartum. One of the main purposes of this study is to collect data related to these outcome as there is limited data in the literature thus we used standard deviation units as a way to determine a reasonable sample size. Assuming the definition of the primary outcomes from above and using a paired t-test for the main analysis with a two-tailed alpha $=0.05$ and power $=0.80$, we are able to detect a difference of as little as 0.30 standard deviation units with 100 patients. Adjusting for potential drop-out or missed data of $10 \%$, we planned recruitment of 110 patients for either change in weight outcomes. To estimate detectable associations between predictors and either change in weight outcomes, we will use correlations since regression coefficients are essentially standardized correlations. So, for correlations between predictors and outcomes, we will have power $=$ 0.80 to detect correlations of 0.28 with 100 patients in a regression model (as described below). In fact, because we will use multivariate regression models to partition the overall variance, we expect to have more power than estimated.

Descriptive statistics will be calculated in the usual way. To estimate the gestational weight change and the post-partum weight change, we will first calculate the change in weight as indicated above and use a standard paired t-test (if the weight change is normally distributed) or a Wilcoxon non-parametric test (if weight change is not normally distributed) to determine if there is a significant change in weight from pre-pregnancy to last prenatal visit preceding delivery weight and from this to six months postpartum. To determine the effect of the various predictors on these outcomes, we will use two approaches to model construction. First, we will use general linear models to model change in weight during gestation or change in weight post-partum, using the definitions for these outcomes from above. Second, we will use a mixed effects model to examine the individual trajectories of change over time, using all of the measures of weight throughout the gestation and postpartum periods. The predictors will be similar for the two analytic approaches, based on the conceptual model in Figure 1. In general we will first investigate the effect of related groups of predictors (such as the behavioral predictors from Table 1) and then combine the resulting significant factors into the final model. With a relatively limited sample size, this will be a feasible approach and potentially more revealing - than a more omnibus variable selection process.

\section{Results}

Demographic characteristics of the study sample $(\mathrm{n}=$ 110) are presented in Table 2. As anticipated, the study population is relatively healthy and consistent with the general population seeking prenatal care in the faculty and resident ambulatory obstetric clinics that constitute the study sites. The mean age is 28.3 years, with a mean pre-pregnancy BMI of $25.7 \mathrm{~kg} / \mathrm{m}$ indicating that a majority of the women were overweight or obese prior to becoming pregnant. The mean gestational age at recruitment was 32.5 weeks. One-third of the cohort is nonwhite, over a quarter are Latina/Hispanic, $15.5 \%$ report a primary language at home other than English, and almost a quarter are foreign born. The majority of participants are multigravida, are high school graduates or have higher levels of education, and work outside of the home. Almost half of the sample reported household financial challenges.

\section{Discussion}

Maternal and childhood obesity are significant public health issues that affect general immediate and long term health of women and children. Women receive more medical attention in pregnancy and the postpartum period than at any other healthful time in their lives; this time is ripe for intervention, especially as women are generally motivated to improve their health for benefit of their offspring. However, this is also a time of significant adaptations as it relates to physical, physiological, hormonal and behavioral changes (including 
Table 2 Cohort demographic characteristics

\begin{tabular}{llll}
\hline Demographic characteristics & Mean & SD & Media \\
\hline Age (years) & 28.3 & 5.1 & 28 \\
Gestational age @baseline study visit (weeks) & 32.5 & 1.8 & 32.6 \\
& N & & \\
Prepregnancy BMI $\left(\mathbf{k g} / \mathbf{m}^{\mathbf{2}}\right)(\mathbf{n}=\mathbf{1 1 0})$ & & & 0.9 \\
Underweight & 1 & & 47.3 \\
Normal & 52 & 29.1 \\
Overweight & 32 & 22.7 \\
Obese & 25 & &
\end{tabular}

Race $(n=93)$

White

$63 \quad 67.7$

Black/African American

Asian

Hawaiian/Pacific Islander

Other

Multiracial

Ethnicity $(\mathrm{n}=99)$

Not Hispanic, Latino or Spanish 7

Hispanic, Latino or Spanish origin $\quad 28$

Non-U.S. born $(\mathrm{n}=99)$

Non-English primary home language $(n=98)$

24.2

Multigravida $(n=108)$

\section{Relationship}

In relationship with father of baby $(n=98)$

Married ( $n=99$ )

Education $(n=99)$

$\leq 8$ th grade

$>8$ th grade, $<$ high school

High school graduate or GED

Trade or technical school after HS

Some college

4 year college degree

Other

Employment $(n=100)$

Working full or part-time outside home

Homemaker

$\begin{array}{ll}65 & 65.5 \\ 15 & 15.0\end{array}$

Household ability to make ends meet $(n=100)$

$\begin{array}{lll}\text { Great difficulty } & 12 & 12.0 \\ \text { Some difficulty } & 36 & 36.0 \\ \text { No difficulty } & 52 & 52.0\end{array}$

Location of care $(n=109)$

Resident practice 
sleep, diet, physical activity, smoking habits), new and different stressors, mood fluctuations, and changes in sociocultural identity and responses from the social environment, along with numerous other weight relevant factors. Excessive pregnancy weight gain and lack of postpartum weight loss $[9,83]$ are associated with maternal obesity which increases the risk of diabetes, cardiac disease [83], some cancers [16], and other health consequences. Maternal obesity and excessive pregnancy weight gain are associated with childhood obesity [3] and its downstream effects which are varied and include metabolic dysfunction, asthma, and behavioral disorders [84]. The critical interplay of socioeconomic issues and weight-related behavioral and other patterns [84] are infrequently or inadequately addressed in weight interventions to improve maternal and offspring health. Comprehensive examination and understanding of behavioral, social, cultural and environmental contexts through a lifespan biopsychosocial model is critical to the design of future interventions with the potential to substantially and effectively improve maternal and child health.

This study is grounded in theory as conceptualized by women's weight trajectories during both pregnancy and the post-partum period, and long-term maternal and child health and well-being, within a lifespan biopsychosocial framework. This study was designed to determine the feasibility of understanding multifactorial influences on maternal pregnancy and postpartum weight trajectories with the goal of gaining critical foundations of knowledge, experience and infrastructure on which to base future intervention research. Large trials recruiting socioeconomically and racially/ethnically diverse women are necessary to identify and analyze moderators and mediators of weight gain and loss as they relate to pregnancy and the postpartum period, and elucidate their effects on maternal and offspring health. We piloted critical variables that are relevant to future large scale studies addressing these issues.

The strengths of this study protocol are several. This is a longitudinal study design that includes a large number of variables and comprehensive assessments. Each of these critical factors individually and/or in aggregate examines different contexts of the biopsychosocial model. We were able to recruit a diverse population of women with $28 \%$ of our subjects being Latina, almost a quarter being non-US born and with approximately 15\% from households speaking primary languages other than English. The study was able to recruit a socioeconomically diverse sample, as evidenced by their range of abilities to meet their household needs. This is critical for analysis of interactions amongst the biologic, psychologic, and social variables. The subject population represents women of childbearing age with more than half being overweight or obese in pregnancy [85], including one out of 5 being obese [86].

A significant strength is the pilot nature of this study performing a pilot acknowledges the importance of determining the feasibility of and acceptance of the design and overall burden to targeted participants. This is critical before embarking on a larger scale endeavor. However, our relatively modest sample size is a limitation. Adequately powering an intervention study with consideration of mediators and modifiers will require a larger sample size and is beyond the scope of this project. Generalizability of study findings may be limited by exclusion of non-English speakers, women with pre-existing physical and mental health conditions, adolescents, and women who did not seek care in early pregnancy.

Evidence of feasibility, acceptability, and consideration of weight influencing factors and behaviors other than diet and physical activity are essential to future epidemiological and intervention studies, including the design of new interventions that optimize appropriate maternal gestational weight gain and subsequent postpartum weight loss. We anticipate that the current study will produce valuable data and insights to help guide application of these findings to interventions targeting maternal health for maternal benefit but also for offspring benefit, thus promoting intergenerational health.

\section{Abbreviations}

BMI: Body mass index; DOHad: Developmental origins of health and disease; EPDS: Edinburgh postnatal depression scale; GWG: Gestational weight gain; HIPAA: Health Insurance Portability and Accountability Act; IOM: Institute of Medicine; NIH: National Institute of Health; PPODS: Pregnancy and postpartum observational determinants study; vWF: von Willebrand's Factor; WHO: World Health Organization.

\section{Competing interests}

The authors declare that they have no competing interests.

\section{Authors' contributions}

TMS, SC and MCR conceived of the study, made substantial contributions to conception and design of the study, drafted the manuscript, have been involved in revising the manuscript critically for important intellectual content. MML, BO, and BB have made substantial intellectual contributions to the conception and design of the study. NNZ made substantial contributions to the acquisition of data for this study and managed collection, processing, and analyses of biologic specimens. KL made substantial contributions to data acquisition, analyses and interpretation. All authors have been involved in reviewing the manuscript critically for important intellectual content, for revising and editing, and have given final approval of the version to be published.

\section{Acknowledgements}

Research reported in this publication was supported by the National Center for Advancing Translational Sciences of the National Institutes of Health under award number UL1TR000161. Dr. Rosal also received funding from NIH/NIMHD (grant \#1 P60 MD006912-02) and the CDC (grant \# U48 DP001933). The content is solely the responsibility of the authors and does not necessarily represent the official views of the $\mathrm{NIH}$. The authors acknowledge the research staff involved in recruitment and retention activities (Laura Robidoux, Jodi Adams Puleo, Cassandra O'Connell), resident physicians whom facilitated data collection related to the delivery time point (Crina Boeras), and staff on whom we relied for administrative support for study activities (Sharon Smith, Gin-Fei Olendzki). 


\section{Author details}

'Department of Obstetrics \& Gynecology, Division of Research, University of Massachusetts Medical School/UMass Memorial Health Care, Memorial Campus - 119 Belmont Street, Worcester, MA 01605, USA. ²Department of Medicine, Program in Molecular Medicine, University of Massachusetts Medical School, Biotech 2 - 373 Plantation Street, Worcester, MA 01605, USA. ${ }^{3}$ Department of Pediatrics, University of Massachusetts Medical School/UMass Memorial Health Care, University Campus - 55 Lake Avenue, Worcester, MA 01655, USA. ${ }^{4}$ Department of Medicine, Division of Preventive and Behaviora Medicine, University of Massachusetts Medical School, University Campus 55 Lake Avenue, Worcester, MA 01655, USA. ${ }^{5}$ Department of Quantitative Health Sciences, University of Massachusetts Medical School, University Campus - 55 Lake Avenue, Worcester, MA 01655, USA.

Received: 11 November 2014 Accepted: 25 February 2015 Published online: 28 March 2015

\section{References}

1. Flegal KM, Carroll MD, Ogden CL, Curtin LR. Prevalence and trends in obesity among US adults, 1999-2008. JAMA. 2010;303(3):235-41.

2. Flegal $\mathrm{KM}$, Carroll MD, Ogden $\mathrm{CL}$, Johnson $\mathrm{CL}$. Prevalence and trends in obesity among US adults, 1999-2000. JAMA. 2002;288(14):1723-7.

3. Institute of Medicine. Weight gain during pregnancy: reexamining the guidelines. Washington, D.C.: The National Academies Press; 2009.

4. Ogden CL, Carroll MD, Curtin LR, McDowell MA, Tabak CJ, Flegal KM. Prevalence of overweight and obesity in the United States, 1999-2004. JAMA. 2006;295(13):1549-55.

5. Moore Simas TA, Liao X, Garrison A, Sullivan GM, Howard AE, Hardy JR. Impact of updated Institute of Medicine guidelines on prepregnancy body mass index categorization, gestational weight gain recommendations, and needed counseling. J Womens Health. 2011;20(6):837-44.

6. Johnson J, Clifton RG, Roberts JM, Myatt L, Hauth JC, Spong CY, et al. Pregnancy outcomes with weight gain above or below the 2009 Institute of Medicine guidelines. Obstet Gynecol. 2013;121(5):969-75

7. Walker LO, Sterling BS, Timmerman GM. Retention of pregnancy-related weight in the early postpartum period: Implications for women's health services. J Obstet Gynecol Neonatal Nurs. 2005;34(4):418-27.

8. Harris HE, Ellison GT, Clement S. Do the psychosocial and behavioral changes that accompany motherhood influence the impact of pregnancy on long-term weight gain? J Psychosom Obstet Gynaecol. 1999;20(2):65-79.

9. Rooney BL, Schauberger CW. Excess pregnancy weight gain and long-term obesity: One decade later. Obstet Gynecol. 2002;100(2):245-52.

10. Linne $Y$, Dye L, Barkeling B, Rossner S. Weight development over time in parous women-the SPAWN study-15 years follow-up. Int J Obes Relat Metab Disord. 2003;27(12):1516-22.

11. Gore SA, Brown DM, West DS. The role of postpartum weight retention in obesity among women: A review of the evidence. Ann Behav Med. 2003:26(2):149-59.

12. Carnethon MR, Loria CM, Hill JO, Sidney S, Savage PJ, Liu K. Risk factors for the metabolic syndrome: The Coronary Artery Risk Development in Young Adults (CARDIA) study, 1985-2001. Diabetes Care. 2004;27(11):2707-15.

13. Norman JE, Bild D, Lewis CE, Liu K, West DS. The impact of weight change on cardiovascular disease risk factors in young black and white adults: The CARDIA study. Int J Obes Relat Metab Disord. 2003;27(3):369-76.

14. Daviglus ML, Liu K, Yan LL, Pirzada A, Manheim L, Manning W, et al. Relation of body mass index in young adulthood and middle age to Medicare expenditures in older age. JAMA. 2004;292(22):2743-9.

15. Truesdale KP, Stevens J, Lewis CE, Schreiner PJ, Loria CM, Cai J. Changes in risk factors for cardiovascular disease by baseline weight status in young adults who maintain or gain weight over 15 years: The CARDIA study. Int Obes (2005). 2006;30(9):1397-407.

16. Boeing H. Obesity and cancer-the update 2013. Best Pract Res Clin Endocrinol Metab. 2013;27(2):219-27.

17. Gaillard R, Durmus B, Hofman A, Mackenbach JP, Steegers EA, Jaddoe WW Risk factors and outcomes of maternal obesity and excessive weight gain during pregnancy. Obesity (Silver Spring). 2013;21(5):1046-55.

18. Oken E, Taveras EM, Kleinman KP, Rich-Edwards JW, Gillman MW. Gestational weight gain and child adiposity at age 3 years. Am J Obstet Gynecol. 2007;196(4):322. e321-328.
19. Kemp MW, Kallapur SG, Jobe AH, Newnham JP. Obesity and the developmental origins of health and disease. J Paediatr Child Health 2012:48(2):86-90

20. Asbee SM, Jenkins TR, Butler JR, White J, Elliot M, Rutledge A. Preventing excessive weight gain during pregnancy through dietary and lifestyle counseling: a randomized controlled trial. Obstet Gynecol. 2009:113(2 Pt 1):305-12.

21. Guelinckx I, Devlieger R, Mullie P, Vansant G. Effect of lifestyle intervention on dietary habits, physical activity, and gestational weight gain in obese pregnant women: a randomized controlled trial. Am J Clin Nutr. 2010;91(2):373-80

22. Jeffries K, Shub A, Walker SP, Hiscock R, Permezel M. Reducing excessive weight gain in pregnancy: a randomised controlled trial. Med J Aust. 2009:191(8):429-33.

23. Kafatos $A G$, Vlachonikolis IG, Codrington CA. Nutrition during pregnancy: the effects of an educational intervention program in Greece. Am J Clin Nutr. 1989;50(5):970-9

24. Kuhlmann AK, Dietz PM, Galavotti C, England LJ. Weight-management interventions for pregnant or postpartum women. Am J Prev Med. 2008;34(6):523-8.

25. Polley BA, Wing RR, Sims CJ. Randomized controlled trial to prevent excessive weight gain in pregnant women. Int J Obes Relat Metab Disord. 2002;26(11):1494-502.

26. Thornton YS, Smarkola C, Kopacz SM, Ishoof SB. Perinatal outcomes in nutritionally monitored obese pregnant women: a randomized clinical trial. J Natl Med Assoc. 2009;101(6):569-77.

27. Amorim Adegboye AR, Linne YM. Diet or exercise, or both, for weight reduction in women after childbirth. Cochrane Database Syst Rev. 2013;7:Cd005627.

28. Dewey KG, Lovelady CA, Nommsen-Rivers LA, McCrory MA, Lonnerdal B. A randomized study of the effects of aerobic exercise by lactating women on breast-milk volume and composition. N Engl J Med. 1994;330(7):449-53.

29. Ferrara $\mathrm{A}$, Hedderson $\mathrm{MM}$, Albright $\mathrm{CL}$, Ehrlich SF, Quesenberry Jr CP, Peng $T$, et al. A pregnancy and postpartum lifestyle intervention in women with gestational diabetes mellitus reduces diabetes risk factors: a feasibility randomized control trial. Diabetes Care. 2011;34(7):1519-25.

30. Lovelady CA, Garner KE, Moreno KL, Williams JP. The effect of weight loss in overweight, lactating women on the growth of their infants. N Engl J Med. 2000;342(7):449-53.

31. McCrory MA, Nommsen-Rivers LA, Mole PA, Lonnerdal B, Dewey KG. Randomized trial of the short-term effects of dieting compared with dieting plus aerobic exercise on lactation performance. Am J Clin Nutr. 1999;69(5):959-67.

32. Kearney $\mathrm{MH}$, Simonelli MC. Intervention fidelity: lessons learned from an unsuccessful pilot study. Appl Nurs Res. 2006;19(3):163-6.

33. Magee L, Hale L. Longitudinal associations between sleep duration and subsequent weight gain: a systematic review. Sleep Med Rev. 2012;16(3):231-41.

34. Knutson KL. Does inadequate sleep play a role in vulnerability to obesity? Am J Hum Biol. 2012;24(3):361-71.

35. Patel SR. Reduced sleep as an obesity risk factor. Obes Rev. 2009;10 Suppl 2:61-8.

36. Wardle J, Chida Y, Gibson EL, Whitaker KL, Steptoe A. Stress and adiposity: a meta-analysis of longitudinal studies. Obesity (Silver Spring). 2011:19(4):771-8.

37. Yau YH, Potenza MN. Stress and eating behaviors. Minerva Endocrinol. 2013;38(3):255-67.

38. Torres SJ, Nowson CA. Relationship between stress, eating behavior, and obesity. Nutrition. 2007;23(11-12):887-94.

39. Insana SP, Montgomery-Downs HE. Sleep and sleepiness among first-time postpartum parents: a field- and laboratory-based multimethod assessment. Dev Psychobiol. 2013;55(4):361-72.

40. Lee KA. Alterations in sleep during pregnancy and postpartum: a review of 30 years of research. Sleep Med Rev. 1998;2(4):231-42.

41. O'Hara MW. Social support, life events, and depression during pregnancy and the puerperium. Arch Gen Psychiatry. 1986;43(6):569-73.

42. Vernon MM, Young-Hyman D, Looney SW. Maternal stress, physical activity, and body mass index during new mothers' first year postpartum. Women Health. 2010;50(6):544-62.

43. Lindberg UB, Leibel RL, Silfverstolpe G, Hirsch J, Bjorntorp P, Rebuffe-Scrive M. Effects of early pregnancy on regional adipose tissue metabolism. Horm Metab Res. 1991;23(1):25-9.

44. Koutsari C, Dumesic DA, Patterson BW, Votruba SB, Jensen MD. Plasma free fatty acid storage in subcutaneous and visceral adipose tissue in postabsorptive women. Diabetes. 2008;57(5):1186-94. 
45. Votruba SB, Mattison RS, Dumesic DA, Koutsari C, Jensen MD. Meal fatty acid uptake in visceral fat in women. Diabetes. 2007;56(10):2589-97.

46. Rebuffe-Scrive M, Enk L, Crona N, Lonnroth P, Abrahamsson L, Smith U, et al. Fat cell metabolism in different regions in women. Effect of menstrual cycle, pregnancy, and lactation. J Clin Invest. 1985;75(6):1973-6.

47. Engel GL. The need for a new medical model: a challenge for biomedicine. Science. 1977;196(4286):129-36.

48. American College of Obstetricians and Gynecologists (ACOG) Committee on Practice Bulletins-Obstetrics. Practice Bulletin No. 137: Gestational Diabetes Mellitus. Obstet Gynecol. 2013;122(2 Pt 1):406-16.

49. American College of Obstetricians and Gynecologists (ACOG) Committee on Practice Bulletins-Obstetrics. Practice Bulletin No. 101: Ultrasonography in pregnancy. Obstet Gynecol. 2009;113(2 Pt 1):451-61.

50. The sixth report of the Joint National Committee on prevention, detection, evaluation, and treatment of high blood pressure. Archives of internal medicine. 1997;157(21):2413-2446.

51. Sturrock ND, Pound N, Peck GM, Soar CM, Jeffcoate WJ. An assessment of blood pressure measurement in a diabetic clinic using random-zero, semi-automated, and 24-hour monitoring. Diabet Med. 1997:14(5):370-5.

52. Huston Presley L, Wong WW, Roman NM, Amini SB, Catalano PM. Anthropometric estimation of maternal body composition in late gestation. Obstet Gynecol. 2000;96(1):33-7.

53. Fattah C, Farah N, Barry S, O'Connor N, Stuart B, Turner MJ. The measurement of maternal adiposity. J Obstet Gynaecol. 2009;29(8):686-9.

54. Heath EM, Morken NW, Campbell KA, Tkach D, Boyd EA, Strom DA. Use of buccal cells collected in mouthwash as a source of DNA for clinical testing. Arch Pathol Lab Med. 2001;125(1):127-33.

55. Machann J, Thamer C, Stefan N, Schwenzer NF, Kantartzis K, Haring HU, et al. Follow-up whole-body assessment of adipose tissue compartments during a lifestyle intervention in a large cohort at increased risk for type 2 diabetes. Radiology. 2010;257(2):353-63.

56. Beaton GH, Milner J, Corey P, McGuire V, Cousins M, Stewart E, et al. Sources of variance in 24-hour dietary recall data: Implications for nutrition study design and interpretation. Am J Clin Nutr. 1979;32:2546-9.

57. Sievert YA, Schakel SF, Buzzard IM. Maintenance of a nutrient database for clinical trials. Control Clin Trials. 1989:10(4):416-25.

58. Matthews CE, Freedson PS, Hebert JR, Stanek 3rd EJ, Merriam PA, Ockene IS. Comparing physical activity assessment methods in the Seasonal Variation of Blood Cholesterol Study. Med Sci Sports Exerc. 2000;32(5):976-84.

59. Matthews CE, DuBose KD, LaMonte M, Tudor-Locke C, Ainsworth BE. Evaluation of a computerized 24-hour physical activity recall (24PAR). [abstract]. Med Sci Sports Exer. 2002:34 Suppl 5:S41.

60. Ainsworth BE, Haskell WL, Whitt MC, Irwin ML, Swartz AM, Strath SJ, et al. Compendium of physical activities: an update of activity codes and MET intensities. Med Sci Sports Exerc. 2000;32(9 Suppl):S498-504.

61. Chasan-Taber L, Schmidt MD, Roberts DE, Hosmer D, Markenson G, Freedson PS. Development and validation of a Pregnancy Physical Activity Questionnaire. Med Sci Sports Exerc. 2004;36(10):1750-60.

62. Infant Feeding Practices Study II: The questionnaires. [http://www.cdc.gov/ ifps/questionnaires.htm]

63. Lakshman RR, Landsbaugh JR, Schiff A, Hardeman W, Ong KK, Griffin SJ. Development of a questionnaire to assess maternal attitudes towards infant growth and milk feeding practices. Int J Behav Nutr Phys Act. 2011;8:35.

64. Buysse DJ, Reynolds 3rd CF, Monk TH, Berman SR, Kupfer DJ. The Pittsburgh Sleep Quality Index: a new instrument for psychiatric practice and research. Psychiatry Res. 1989;28(2):193-213.

65. Field T, Diego M, Hernandez-Reif M, Figueiredo B, Schanberg S, Kuhn C. Sleep disturbances in depressed pregnant women and their newborns. Infant Behav Dev. 2007;30(1):127-33.

66. Coo S, Milgrom J, Kuppens P, Cox P, Trinder J. Exploring the association between maternal mood and self-reports of sleep during the perinatal period. J Obstet Gynecol Neonatal Nurs. 2014;43(4):465-77.

67. Behavioral Risk Factor Surveillance System. [http://www.cdc.gov/brfss/ questionnaires.htm\#english]

68. Spielberger CD. Manual for the state-trait anxiety inventory. Palo Alto, CA: Consulting Psychologists Press; 1983.

69. Cox JL, Holden JM, Sagovsky R. Detection of postnatal depression. Development of the 10-item Edinburgh Postnatal Depression Scale. Br J Psychiatry. 1987;150:782-6.
70. Jadresic E, Araya R, Jara C. Validation of the Edinburgh Postnatal Depression Scale (EPDS) in Chilean postpartum women. J Psychosom Obstet Gynaecol. 1995;16(4):187-91.

71. Cohen S, Kamark T, Mermelstein R. A global measure of perceived stress. J Health Soc Behav. 1983;24(4):385-96.

72. Sandman CA, Davis EP, Buss C, Glynn LM. Exposure to prenatal psychobiological stress exerts programming influences on the mother and her fetus. Neuroendocrinology. 2012;95(1):7-21.

73. Jenkinson C, Layte $R$, Jenkinson D, Lawrence $K$, Petersen S, Paice C, et al. A shorter form health survey: can the SF-12 replicate results from the SF-36 in longitudinal studies? J Public Health Med. 1997;19(2):179-86.

74. Lyubomirsky S, Lepper HS. A measure of subjective happiness: preliminary reliability and construct validation. Soc Indic Res. 1999:46:137-55.

75. Palmer JL, Jennings GE, Massey L. Development of an assessment form: attitude toward weight gain during pregnancy. J Am Diet Assoc. 1985;85(8):946-9.

76. Karlsson J, Persson LO, Sjostrom L, Sullivan M. Psychometric properties and factor structure of the Three-Factor Eating Questionnaire (TFEQ) in obese men and women. Results from the Swedish Obese Subjects (SOS) study. Int J Obes Relat Metab Disord. 2000;24(12):1715-25.

77. White MA, Grilo CM. Psychometric properties of the Food Craving Inventory among obese patients with binge eating disorder. Eat Behav. 2005:6(3):239-45.

78. Sherbourne CD, Stewart AL. The MOS social support survey. Soc Sci Med. 1991;32(6):705-14.

79. Surkan PJ, Ryan LM, Carvalho Vieira LM, Berkman LF, Peterson KE. Maternal social and pyschological conditions and physical growth in low-income children in Piaui, Northeast Brazil. Soc Sci Med. 2007;64(2):375-88.

80. Wadhwa PD, Dunkel-Schetter C, Chicz-DeMet A, Porto M, Sandman CA. Prenatal psychosocial factors and the neuroendocrine axis in human pregnancy. Psychosom Med. 1996;58(5):432-46.

81. Collins NL, Dunkel-Schetter C, Lobel M, Scrimshaw SC. Social support in pregnancy: psychosocial correlates of birth outcomes and postpartum depression. J Pers Soc Psychol. 1993;65(6):1243-58.

82. Rasmussen $\mathrm{KM}$, Yaktine $\mathrm{AL}$, editors. Weight gain during pregnancy: Re-examining the guidelines-Prepublication copy. Washington, DC: National Academy Press; 2009.

83. Rooney BL, Schauberger CW, Mathiason MA. Impact of perinatal weight change on long term obesity and obesity-related illnesses. Obstet Gynecol Annu. 2005;106:1349-56.

84. O'Reilly JR, Reynolds RM. The risk of maternal obesity to the long-term health of the offspring. Clin Endocrinol (Oxf). 2013;78(1):9-16.

85. Committee ACOG. opinion no. 549: obesity in pregnancy. Obstet Gynecol. 2013;121(1):213-7.

86. Chu SY, Callaghan WM, Bish CL, D'Angelo D. Gestational weight gain by body mass index among U.S. women delivering live births, 2004-2005: fueling future obesity. Am J Obstet Gynecol. 2009;200:271. e271-277.

\section{Submit your next manuscript to BioMed Central and take full advantage of:}

- Convenient online submission

- Thorough peer review

- No space constraints or color figure charges

- Immediate publication on acceptance

- Inclusion in PubMed, CAS, Scopus and Google Scholar

- Research which is freely available for redistribution 